\title{
Comparison of arteriosclerotic indicators in patients with ischemic stroke: ankle-brachial index, brachial-ankle pulse wave velocity and cardio-ankle vascular index
}

\begin{abstract}
Naoki Saji ${ }^{1,2}$, Kazumi Kimura ${ }^{1,3}$, Yoshiki Yagita ${ }^{1}$, Toshitaka Kawarai ${ }^{2}$, Hirotaka Shimizu ${ }^{2}$ and Yasushi Kita ${ }^{2}$
The ankle-brachial index (ABI), brachial-ankle pulse wave velocity (baPWV) and cardio-ankle vascular index (CAVI) are surrogate markers of arteriosclerosis. However, their roles in patients with acute ischemic stroke remain unclear. From October 2003 to September 2011, we enrolled patients with arteriosclerotic ischemic stroke (AIS) exhibiting large infarcts attributed to large-artery atherosclerosis (LAA) or deep subcortical infarcts (mainly lacunar infarcts) attributed to small-artery disease (SAD). Outpatients without a history of stroke served as controls (CTL). We divided the study period into two terms and assessed patients using two different oscillometric devices (Form PWV/ABI, Omron Colin; and VaSera VS-1500, Fukuda Denshi) in each term. One-way analysis of variance and age- and sex-adjusted analysis of covariance were used to compare the three groups. We analyzed 842 patients. The ABI was significantly lower in the LAA $(n=102)$ group than in the SAD $(n=280)$ and CTL $(n=460)$ groups. The baPWV was significantly higher in the LAA and SAD groups than in the CTL group. The CAVI gradually increased in the order of CTL, SAD and LAA. The cutoff values of baPWV and CAVI for detection of AIS were $18.3 \mathrm{~m} \mathrm{~s}^{-1}$ (odds ratio (OR): $6.09,95 \%$ confidence interval $\left.(\mathrm{CI}): 3.97-9.62, P<0.01\right)$ and $9.5(0 R: 1.44,95 \% \mathrm{Cl}: 1.24-1.70$, $\boldsymbol{P}<0.001$ ), respectively. Among the three indicators, a lower ABI indicated advanced atherosclerosis associated with LAA, and an increased baPWV more closely indicated AIS. An increased CAVI may indicate the degree of vessel stiffness due to arteriosclerosis.
\end{abstract}

Hypertension Research (2015) 38, 323-328; doi:10.1038/hr.2015.8; published online 26 February 2015

Keywords: ankle-brachial index; arterial stiffness; cardio-ankle vascular index; pulse wave velocity; stroke

\section{INTRODUCTION}

Arterial stiffness has been monitored by pulse wave velocity (PWV) parameters such as the carotid-femoral PWV and brachial-ankle PWV (baPWV). ${ }^{1-4}$ Arterial stiffness is associated with atherosclerosis and is a surrogate marker for both cardiovascular disease and cerebrovascular disease..$^{3-10}$ The cardio-ankle vascular index (CAVI), a novel surrogate marker of arterial stiffness that can be calculated using the PWV independently of blood pressure during a single measurement, was recently studied with respect to its role in the evaluation of arteriosclerosis. ${ }^{11-16}$ Our earlier studies have shown that these indicators are associated with cerebrovascular diseases, such as silent lacunar infarcts (SLI), white matter disease (WMD) and acute subcortical infarcts, which are associated with cerebral small vessel disease ${ }^{8-11}$ and often coexist. ${ }^{17}$ Likewise, the ankle-brachial index (ABI) is also used to assess advanced atheromatous changes in large arteries, such as those affected by peripheral artery disease, and is associated with cerebrovascular disease. ${ }^{4,18-20}$

Previous studies have revealed that the ABI and PWV are associated with intracerebral atherosclerosis. ${ }^{21-23}$ However, such an association regarding the CAVI and comparison among patients with and without stroke has not been fully investigated. Furthermore, assessing arterial stiffness in patients with acute ischemic stroke may be problematic, because these patients have high blood pressure, which affects PWV. 15,21,23 Assessment of the CAVI among patients with and without ischemic stroke may highlight the arteriosclerotic differences from the viewpoint of vessel stiffness, because the CAVI can be calculated independently of blood pressure during a single measurement.

The aim of the present study was to compare the distribution of the $\mathrm{ABI}, \mathrm{baPWV}$ and CAVI in patients with ischemic stroke attributed to arteriosclerosis with those in patients without a history of stroke.

\footnotetext{
${ }^{1}$ Department of Stroke Medicine, Kawasaki Medical School, Okayama, Japan; ${ }^{2}$ Department of Neurology, Hyogo Brain and Heart Center at Himeji, Hyogo, Japan and ${ }^{3}$ Department of Neurological Science, Nippon Medical School Graduate School of Medicine, Tokyo, Japan

Correspondence: Dr N Saji, Department of Stroke Medicine, Kawasaki Medical School, 577 Matsushima, Kurashiki, Okayama 701-0192, Japan.

E-mail: sajink@nifty.com

Received 18 July 2014; revised 19 November 2014; accepted 11 December 2014; published online 26 February 2015
} 


\section{MATERIALS AND METHODS}

\section{Study design}

This was a hospital-based, retrospective, cross-sectional study approved by the Institutional Review Board of Hyogo Brain and Heart Center at Himeji. This study was performed from October 2003 to September 2011. Informed consent was obtained from the patients or their family members. This study complied with the Declaration of Helsinki and is registered with UMIN Clinical Trials Registry (UMIN000014147).

\section{Participants}

We enrolled consecutive patients with acute ischemic stroke admitted to the Hyogo Brain and Heart Center at Himeji within $48 \mathrm{~h}$ after stroke onset. Patients with acute ischemic stroke underwent a standard systemic investigation, including blood tests, chest radiograph, electrocardiography, carotid ultrasonography, transthoracic echocardiography and 24-h Holter electrocardiography. The type of ischemic stroke in each patient was classified as one of the following traditional stroke subtypes described in the Trial of Org 10172 in Acute Stroke Treatment classification: (1) large-artery atherosclerosis (LAA), (2) lacunar infarcts, (3) cardioembolic infarcts, (4) stroke of other determined etiology, and (5) stroke of undetermined etiology. ${ }^{24,25}$ LAA was defined as the presence of cortical or cerebellar dysfunction and a large infarct (initial diameter of $\geqslant 1.5 \mathrm{~cm}$ ) attribute to $\geqslant 50 \%$ stenosis of an appropriate intracranial or extracranial artery. Lacunar infarcts were defined as the presence of a clinical lacunar syndrome attributed to a deep small subcortical infarct (initial diameter of $<1.5 \mathrm{~cm}$ ) without $\geqslant 50 \%$ stenosis of an appropriate artery. Patients with a clinical lacunar syndrome attributed to a deep subcortical infarct (initial diameter of $\geqslant 1.5 \mathrm{~cm}$ ) without $\geqslant 50 \%$ stenosis of an appropriate artery were combined with lacunar infarcts and categorized as having small-artery diseases $(\mathrm{SAD})$, because infarct size on magnetic resonance diffusion-weighted imaging tends to be temporarily larger during the acute phase. ${ }^{8}$ LAA and SAD were categorized as arteriosclerotic ischemic stroke (AIS).

The inclusion criterion in the present study was the presence of LAA or SAD. Patients were excluded if they had recurrent stroke, atrial fibrillation, atrial flutter or peripheral arterial disease (defined as $\mathrm{ABI}<0.9$ as described below), because these factors might affect the assessment of arterial stiffness. ${ }^{10}$

\section{Control group}

Outpatients who met all of the following criteria were included in the control group (CTL): (1) visited the clinic in the Department of Neurology at the Hyogo Brain and Heart Center at Himeji to request medical evaluation of possible cerebrovascular disease; (2) completed magnetic resonance imaging (MRI) of the brain, carotid ultrasonography and measurement of arterial stiffness; (3) had no history of stroke, transient ischemic attack or dementia; (4) exhibited no abnormalities on neurological examination; and (5) exhibited no atrial fibrillation, atrial flutter or peripheral arterial disease. The details of the clinical characteristics of these outpatients were separately analyzed and have been described elsewhere. ${ }^{9-11}$

\section{Magnetic resonance imaging \\ Patients underwent brain MRI (Intera 1.5-T MRI system or Achieva 3-T MRI system; Philips Medical Systems, Best, The Netherlands), including diffusion- weighted imaging, fluid-attenuated inversion recovery (FLAIR) imaging and T2-weighted imaging. One trained neurologist (NS) assessed all of the MRI images and was blind to all other patient data. SLI was defined as a focal lesion of at least $3 \mathrm{~mm}$ in diameter, with hyperintensity on T2-weighted images and hypointensity on FLAIR images. ${ }^{8-11}$ WMD was defined as an irregular periventricular hyperintensity (Fazekas grade $\geqslant 3$ ) and/or early confluent or confluent separate deep white matter hyperintense lesions (Fazekas grade $\geqslant 2$ ) on FLAIR images. ${ }^{8-11}$}

\section{Carotid ultrasonography}

The far wall common carotid artery intima-media thickness (CCA-IMT) was measured using images acquired by high-resolution B-mode ultrasonography with a 7.5-MHz linear array transducer (Aplio XG SSA-790A, Toshiba Medical, Tokyo, Japan). Three determinations of the intima-media thickness were performed at the thickest point: the maximum CCA-IMT and two adjacent points $\left(1 \mathrm{~cm}\right.$ upstream and $1 \mathrm{~cm}$ downstream from this site). ${ }^{26}$ These three determinations were averaged to obtain the mean CCA-IMT. The mean CCAIMT was determined on both the left and right sides, and the larger value was used for analyses. ${ }^{9-11,27}$

\section{Assessment of arterial stiffness}

We assessed arterial stiffness 1 week after stroke onset using a previously described oscillometric method. ${ }^{1-3,6-15,23}$ Briefly, patients were placed in the supine position and rested for $5 \mathrm{~min}$ before measurements. Electrocardiographic electrodes were placed on both wrists, cuffs were wrapped around both ankles and the brachial regions of both forearms and the heart sounds were monitored. Trained laboratory technicians blind to the patient data measured the arterial stiffness. We divided the study period into two terms and assessed patients using two oscillometric devises in each term. The ABI and baPWV were measured using the Form PWV/ABI (Omron Colin, Tokyo, Japan) in the former term, and the ABI and CAVI were measured using the VaSera VS-1500 (Fukuda Denshi, Tokyo, Japan) in the latter term (Figure 1). The ABI was analyzed in one cohort comprising all patients recruited throughout the study period, because the ABI can be calculated by the same formula using both devices. A lower ABI of $<0.9$ was considered indicative of peripheral arterial disease. ${ }^{4}$ The schema of the ABI, baPWV and CAVI are provided in the online (Supplementary Figure S1).

\section{Risk factors}

The definitions of the risk factors described below were in accordance with those used in our previous studies..$^{8-11}$ Hypertension was defined as a systolic blood pressure of $\geqslant 140 \mathrm{~mm} \mathrm{Hg}$, a diastolic blood pressure of $\geqslant 90 \mathrm{~mm} \mathrm{Hg}$ and/ or the use of antihypertensive agents. Dyslipidemia was defined as a serum total cholesterol level of $\geqslant 220 \mathrm{mg} \mathrm{dl}^{-1}$ and/or the use of statins. Diabetes mellitus was defined as a hemoglobin Alc level of $\geqslant 6.5 \%$ and/or the use of oral hypoglycemic agents or insulin and/or a serum fasting blood glucose level of $\geqslant 126 \mathrm{mg} \mathrm{dl}^{-1}$. Ischemic heart disease was defined as a history of cardiologistdiagnosed angina pectoris or evidence of prior myocardial infarction or a prior coronary revascularization procedure (percutaneous coronary intervention or coronary artery bypass surgery). Current smoking habits were recorded.

\section{Statistical analysis}

Continuous variables are expressed as mean and s.d. and were compared using the unpaired Student's $t$-test. Variables exhibiting skewed distributions are expressed as median and interquartile range and were compared using the Wilcoxon rank-sum test. Categorical variables are expressed as frequencies and percentages and were compared using the chi-squared test. Data were analyzed using one-way analysis of variance and post hoc tests (Tukey's honest significant difference test) and analysis of covariance adjusted for age and sex as well as for age, sex and systolic blood pressure for multiple group comparisons. Receiver operating characteristic curve analysis with the area under the receiver

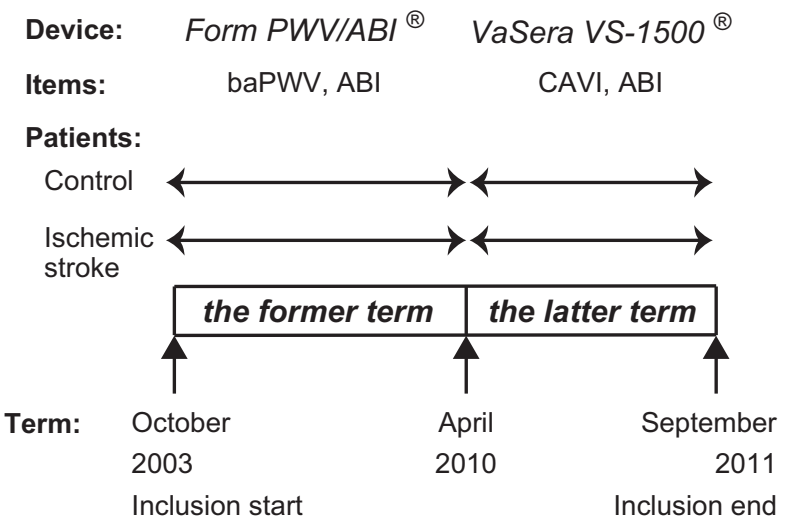

Figure 1 Schema of the study design. 
operating characteristic curve (AUC) was performed to determine the cutoff values of both baPWV and CAVI for the presence of AIS. We did not assess the cutoff values of either ABI or CCA-IMT because an ABI of $<0.9$ and a CCAIMT of $\geqslant 1.1 \mathrm{~mm}$ (otherwise $\geqslant 1.0 \mathrm{~mm}$ ) are widely accepted cutoff values regarding cardiovascular, cerebrovascular and peripheral vascular disease. ${ }^{4,28} \mathrm{~A}$ two-tailed $P$-value of $<0.05$ was considered to indicate statistical significance. Odds ratios (ORs) are presented with 95\% confidence intervals (95\% CIs). Data were analyzed using the JMP 11.2 software package (SAS Institute, Cary, NC, USA).

\section{RESULTS}

\section{Patient recruitment}

During the study period, 1380 patients with acute ischemic stroke were admitted to the Hyogo Brain and Heart Center at Himeji within $48 \mathrm{~h}$ after stroke onset. We excluded 998 of these 1380 patients (Figure 2). Hence, 382 patients with first-ever AIS were enrolled (SAD, $n=280$; LAA, $n=102$ ). The percentages of ischemic stroke subtypes in the former and latter terms were similar (SAD vs. LAD: $73.7 \%$ vs. $26.3 \%$ and $71.4 \%$ vs. $28.6 \%$, respectively). During the same period, 460 outpatients were enrolled and served as the CTL group (240 in former term and 220 in latter term). Therefore, we analyzed 842 patients in the present study.

\section{Patient characteristics}

Patients with AIS had a significantly higher blood pressure, more risk factors and a higher prevalence of SLI and WMD than did patients in the CTL group (Supplementary Table S1). No significant difference in the CCA-IMT was noted between patients with and without AIS. Patients with SAD had a higher blood pressure and a higher prevalence of SLI and WMD than did patients with LAA

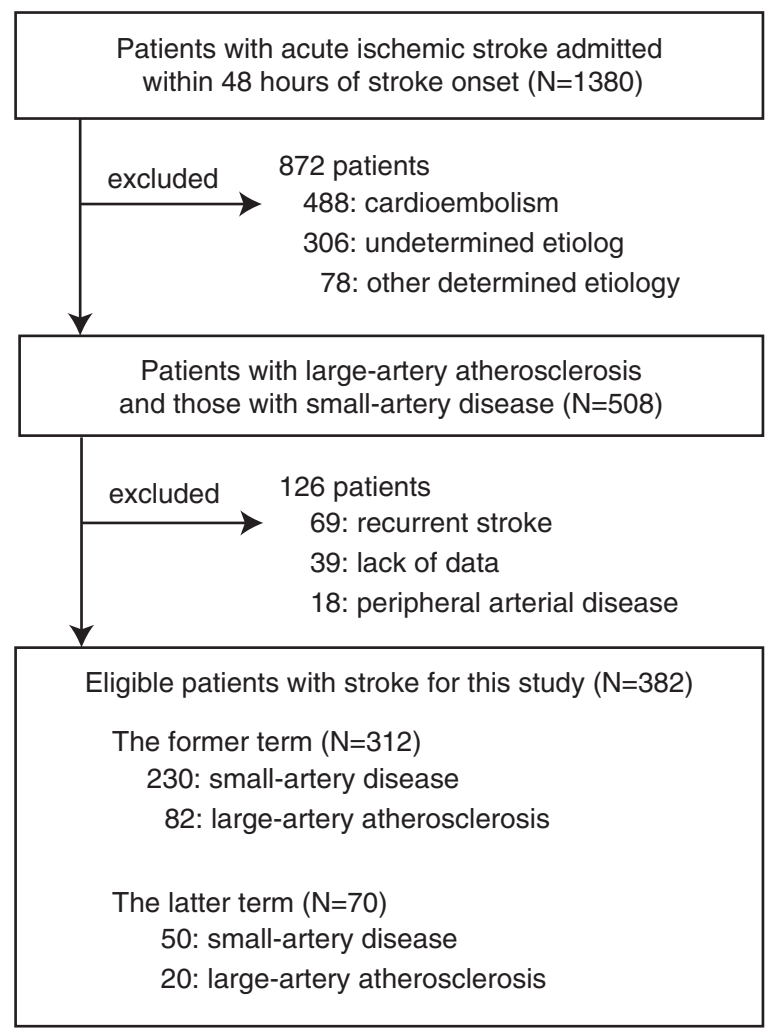

Figure 2 Flow chart of patients included in the study.
(Supplementary Table S2). The CCA-IMT was greater in patients with LAA than in those with SAD.

\section{Assessment of arterial stiffness}

Both the baPWV and CAVI in patients with AIS were significantly higher than those in patients in the CTL group (baPWV, 20.8 vs. $17.1 \mathrm{~m} \mathrm{~s}^{-1}$; and CAVI, 10.0 vs. 9.1, respectively; $P<0.0001$ for both) (Supplementary Table S1). Although the CAVI in patients with SAD was lower than that in patients with LAA (9.9 vs. 10.7, respectively; $P=0.198$ ), the baPWV in patients with SAD was not different from that in patients with LAA ( $20.8 v$ s. $21.0 \mathrm{~m} \mathrm{~s}^{-1}$, respectively; $\left.P=0.661\right)$ (Supplementary Table S2).

Table 1 shows the distribution of the ABI, baPWV and CAVI among the three groups (LAD, SAD and CTL) assessed by analysis of variance (Figure 3). Patients with LAA and those with SAD had a higher baPWV than did patients in the CTL group. The CAVI increased gradually in the order of CTL, SAD and LAA. The ABI in patients in the LAA group was significantly lower than that in patients in the SAD and CTL groups. Adjusted analysis of covariance showed statistically significant relationships identical to those shown in the analysis of variance (Table 2). The baPWV in patients with SAD increased to a level identical to or slightly higher than that in patients with LAA after adjustment for age and sex.

\section{Cutoff baPWV and CAVI values}

Age- and sex-adjusted multivariable logistic regression analysis showed that both the baPWV (OR: 1.28, 95\% CI: 1.21-1.35, $P<0.001$ ) and CAVI (OR: 1.53, 95\% CI: $1.28-1.85, P<0.001)$ were independently associated with the presence of AIS. The baPWV and CAVI cutoff values for the detection of AIS were $18.3 \mathrm{~m} \mathrm{~s}^{-1}$ (69\% sensitivity, $67 \%$ specificity, $\quad$ AUC $=0.72)$ and $9.5 \quad(69 \%$ sensitivity, $65 \%$ specificity, AUC $=0.71$ ), respectively (Supplementary Figures S2 and S3).

\section{DISCUSSION}

The main finding of the present study was that both the baPWV and CAVI were higher in patients with AIS than in those without AIS. The ABI in patients with LAA was the lowest among the three groups. Furthermore, the CAVI gradually increased in the order of patients without ischemic stroke, those with SAD and those with LAA, although the baPWV in patients with SAD increased to the same or slightly higher level as that in patients with LAA.

This study highlights the results of a multimethodological assessment of the ABI, baPWV and CAVI between patients with AIS and those without a history of stroke. Previous studies have demonstrated that both the baPWV and CAVI are useful surrogate markers of cerebral small vessel disease and cerebral arteriosclerosis $^{8-11,16,23,25,29-33}$ and that the ABI is useful in the assessment of atherosclerosis and risk of stroke. ${ }^{18,19}$ Our results are in line with these studies. It is not unexpected that both the baPWV and CAVI indicated arteriosclerosis in patients with AIS. However, these indicators showed slightly different patterns between the SAD and LAA groups. This discrepancy might be explained by the different clinical meanings of arteriosclerosis between the two groups of patients.

We speculated that the increase in the baPWV to the same or slightly higher level in patients with SAD and those with LAA resulted from various mechanisms, such as high blood pressure, vascular endothelial dysfunction, blood-brain barrier failure or microcirculatory impairment in addition to vessel stiffness. ${ }^{8,9,13,25,33,34}$ Patients 
Table 1 Comparison among patients without ischemic stroke (CTL), those with small-artery disease (SAD) and those with large-artery atherosclerosis (LAA)

\begin{tabular}{|c|c|c|c|c|}
\hline & $\begin{array}{c}C T L \\
\mathrm{n}=460\end{array}$ & $\begin{array}{c}S A D \\
\mathrm{n}=280\end{array}$ & $\begin{array}{c}L A A \\
\mathrm{n}=102\end{array}$ & $P$ \\
\hline \multicolumn{5}{|l|}{ Demography } \\
\hline Age, years & $69 \pm 9$ & $69 \pm 11$ & $71 \pm 10$ & 0.050 \\
\hline \multicolumn{5}{|l|}{ Physical status } \\
\hline $\mathrm{PP}, \mathrm{mm} \mathrm{Hg}$ & $55(46-63)$ & $61(49-72)$ & $61(52-72)$ & $<0.001$ \\
\hline SLI, $n(\%)$ & $96(21)$ & $146(52)$ & $33(32)$ & $<0.001$ \\
\hline WMD, $n(\%)$ & $184(40)$ & $169(60)$ & $53(52)$ & $<0.001$ \\
\hline \multicolumn{5}{|l|}{ Risk factors } \\
\hline Hypertension, $n$ (\%) & $345(75)$ & $245(88)$ & $85(83)$ & $<0.001$ \\
\hline \multicolumn{5}{|c|}{ Assessment of arteriosclerosis } \\
\hline CCA-IMT, mm & $1.5(1.1-2.0)$ & $1.4(1.1-1.8)$ & $1.6(1.3-2.3)$ & 0.006 \\
\hline $\mathrm{ABI}$ & $1.11(1.07-1.16)$ & $1.11(1.05-1.17)$ & $1.07(1.01-1.12)$ & $<0.001$ \\
\hline baPWV, $\mathrm{ms}^{-1}$ & $17.1(14.7-19.9)$ & $20.8(17.5-25.0)$ & $21.0(18.4-24.4)$ & $<0.001$ \\
\hline CAVI & $9.1(8.1-9.8)$ & $9.9(9.2-11.2)$ & $10.7(9.2-12.3)$ & $<0.001$ \\
\hline
\end{tabular}

Abbreviations: ABI, ankle-brachial index; baPWV, brachial-ankle pulse wave velocity; CAVI, cardio-ankle vascular index; CCA-IMT, common carotid artery intima-media thickness; DBP, diastolic blood pressure; DM, diabetes mellitus; IHD, ischemic heart disease; PP, pulse pressure (SBP-DBP); SBP, systolic blood pressure; SLI, silent lacunar infarction; WMD, white matter disease. Data are presented as mean \pm s.d., median (interquartile range) or $n(\%)$.

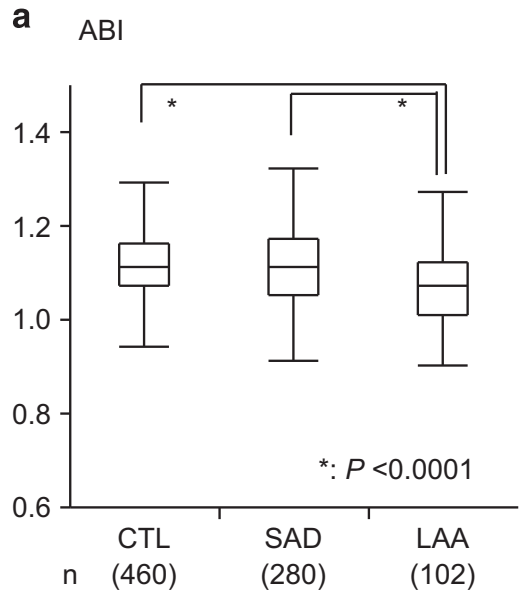

b $\operatorname{baPWV}(\mathrm{m} / \mathrm{s})$

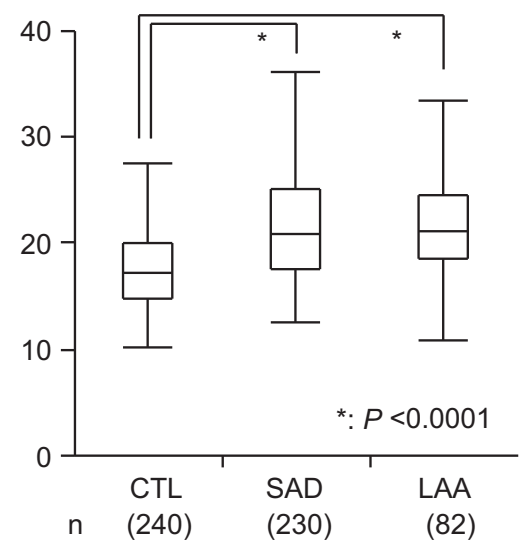

c CAVI

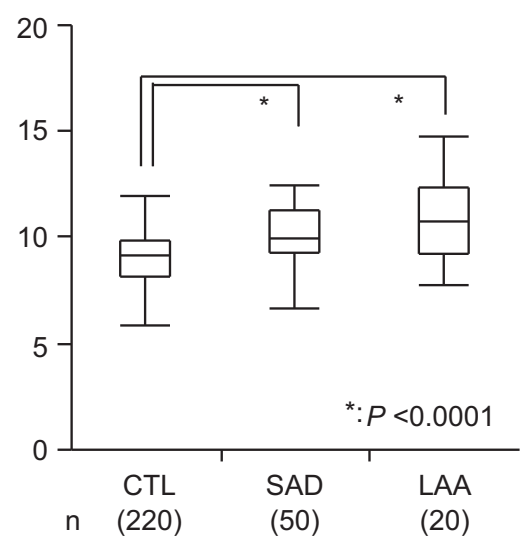

Figure 3 Associations among the (a) ABI, (b) baPWV, and (c) CAVI in the three groups of patients: those without stroke (CTL), those with ischemic stroke attributed to small-artery disease (SAD), and those with ischemic stroke attributed to large-artery atherosclerosis (LAA).

with acute ischemic stroke usually have high blood pressure. This may induce an increase in the baPWV beyond that of actual arteriosclerosis. We previously reported that $\mathrm{SAD}$ in association with progressive neurological deficits was independently associated with a high baPWV. ${ }^{8}$ Likewise, patients with SAD had a higher carotid-femoral PWV than did patients with LAA. ${ }^{25}$ These findings support our concept. Furthermore, we previously showed that the cutoff value of baPWV was $\geqslant 18 \mathrm{~m} \mathrm{~s}^{-1}$ for the presence of progressive neurological deficits, ${ }^{8} \mathrm{SLI}^{9}$ and WMD. ${ }^{10}$ In the present study, we also showed that the cutoff value of baPWV was $\geqslant 18 \mathrm{~m} \mathrm{~s}^{-1}$ as an indicator of the presence of AIS. These cutoff values are in accordance with those of cardiovascular diseases in a recent guideline for noninvasive vascular function testing. ${ }^{1}$

The CAVI may reflect the degree of vessel stiffness, ranging from small-vessel arteriosclerosis to large-artery atheromatous disease, and may allow for a straightforward assessment of vessel stiffness. The 
Table 2 Age- and sex-adjusted differences in ABI, baPWV and CAVI in patients without and those with arteriosclerotic ischemic stroke

\begin{tabular}{|c|c|c|c|c|c|}
\hline & $C T L$ & $S A D$ & $\angle A A$ & $P$ & RMSE \\
\hline \multicolumn{6}{|l|}{$A B I$} \\
\hline Model 1 & $1.11 \pm 0.004$ & $1.11 \pm 0.005$ & $1.07 \pm 0.008^{a}$ & $<0.0001$ & 0.083 \\
\hline \multicolumn{6}{|l|}{ baPWV } \\
\hline Model 1 & $17.7 \pm 0.3$ & $21.6 \pm 0.3^{b}$ & $21.1 \pm 0.5^{b}$ & $<0.0001$ & 0.406 \\
\hline \multicolumn{6}{|l|}{ CAVI } \\
\hline Model 1 & $9.2 \pm 0.1$ & $10.3 \pm 0.2^{c}$ & $10.5 \pm 0.4^{c}$ & $<0.0001$ & 1.600 \\
\hline Model 2 & $9.2 \pm 0.1$ & $10.2 \pm 0.2^{c}$ & $10.5 \pm 0.4^{c}$ & $<0.0001$ & 1.570 \\
\hline
\end{tabular}

Abbreviations: ABI, ankle-brachial index; baPWV, brachial-ankle pulse wave velocity; CAVI, cardio-ankle vascular index; CTL, control (patients without ischemic stroke); LAA, large-artery atherosclerosis; RMSE, root mean square error; SAD, small-artery disease.

Data are presented as mean \pm s.e.

Model 1: Analysis of covariance adjusted for age and sex.

Model 2: Analysis of covariance adjusted for age, sex and systolic blood pressure.

asignificantly higher than both SAD and CTL.

bSignificantly higher than CTL.

'Significantly higher than CTL.

cutoff value of CAVI for the presence of carotid arteriosclerosis in a general population was $\geqslant 8,35$ and this value may indicate an early stage of systemic arteriosclerosis. In the present study, the median CAVI in patients without AIS, with SAD and with LAA was 9, 10 and 11 , respectively. Our results are in concordance with those of previous studies in which a CAVI of $\geqslant 9$ was the cutoff point for the presence of arteriosclerotic diseases, such as coronary artery disease ${ }^{12,36}$ and silent cerebrovascular disease. ${ }^{11}$ However, the small number of recruited patients with AIS in the present study may have led to the lack of a statistically significant difference in the CAVI between patients with SAD and those with LAA.

The ABI in patients with LAA was significantly lower, although there were no significant differences between patients in the SAD and CTL groups. Because an ABI of $<0.9$ indicates $>50 \%$ stenosis between the aorta and the distal leg arteries, this result is reasonable ${ }^{4}$ and in line with that of a previous report. ${ }^{18}$

There are several limitations of this study. Selection bias was possible because this was a cross-sectional study performed in a single hospital-based cohort. The number of patients recruited in the second term was limited. The carotid plaque score ${ }^{37}$ was not assessed. We could not simultaneously measure both the baPWV and CAVI in the same patients; either the baPWV or CAVI was measured in each patient because the medical expense insurance in Japan is restricted to the use of one or the other for assessment of arterial stiffness. According to the recent guideline, ${ }^{1}$ baPWV has been confirmed as a noninvasive vascular function parameter. However, the CAVI has not yet been confirmed as a robust surrogate marker because of insufficient evidence. This issue will need to be addressed in future studies.

In conclusion, the ABI, baPWV and CAVI are differential surrogate markers for arteriosclerosis in patients with AIS. In particular, a lower $\mathrm{ABI}$ indicates advanced atherosclerosis associated with LAA, and an increased baPWV more closely indicates AIS. Meanwhile, an increased CAVI may indicate the degree of vessel stiffness due to arteriosclerosis.

\section{CONFLICT OF INTEREST}

The authors declare no conflict of interest.

\section{ACKNOWLEDGEMENTS}

This study was partially funded by The Kawasaki Foundation for Medical Science and Medical Welfare. Dr Saji has received research grants from The Kawasaki Foundation for Medical Science and Medical Welfare and Grants-inAid for Scientific Research (No. 26870765) from the Japan Society for the Promotion of Science.

1 Yamashina A. Guidelines for non-invasive vascular function test (JCS 2013). Circ J 2013 (in press).

2 Yamashina A, Tomiyama H, Takeda K, Tsuda H, Arai T, Hirose K, Koji Y, Hori S, Yamamoto $Y$. Validity, reproducibility, and clinical significance of noninvasive brachialankle pulse wave velocity measurement. Hypertens Res 2002; 25: 359-364.

3 Tomiyama H, Yamashina A, Arai T, Hirose K, Koji Y, Chikamori T, Hori S, Yamamoto Y, Doba N, Hinohara S. influences of age and gender on results of noninvasive brachialankle pulse wave velocity measurement-a survey of 12517 subjects. Atherosclerosis 2003; 166: 303-309.

4 Perk J, De Backer G, Gohlke H, Graham I, Reiner Z, Verschuren WM, Albus C, Benlian P, Boysen G, Cifkova R, Deaton C, Ebrahim S, Fisher M, Germano G, Hobbs R, Hoes A, Karadeniz S, Mezzani A, Prescott E, Ryden L, Scherer M, Syvänne M, Scholte Op Reimer WJ, Vrints C, Wood D, Zamorano JL, Zannad F. Fifth Joint Task Force of the European Society of Cardiology and Other Societies on Cardiovascular Disease Prevention in Clinical Practice; European Association for Cardiovascular Prevention and Rehabilitation. European Guidelines on cardiovascular disease prevention in clinical practice (version 2012): The Fifth Joint Task Force of the European Society of Cardiology and Other Societies on Cardiovascular Disease Prevention in Clinical Practice (constituted by representatives of nine societies and by invited experts). Atherosclerosis 2012; 223: 1-68.

5 Vlachopoulos C, Aznaouridis K, Terentes-Printzios D, loakeimidis N, Stefanadis C. Prediction of cardiovascular events and all-cause mortality with brachial-ankle elasticity index: a systematic review and meta-analysis. Hypertension 2012; 60: 556-562.

6 Ben-Shlomo Y, Spears M, Boustred C, May M, Anderson SG, Benjamin EJ, Boutouyrie P, Cameron J, Chen CH, Cruickshank JK, Hwang SJ, Lakatta EG, Laurent S, Maldonado J, Mitchell GF, Najjar SS, Newman AB, Ohishi M, Pannier B, Pereira T, Vasan RS, Shokawa T, Sutton-Tyrell K, Verbeke F, Wang K, Webb DJ, Willum Hansen T, Zoungas S, McEniery CM, Cockcroft JR, Wilkinson IB. Aortic pulse wave velocity improves cardiovascular event prediction: an individual participant metaanalysis of prospective observational data from 17,635 subjects. J Am Coll Cardiol 2014; 63: 636-646.

7 Kim J, Song TJ, Song D, Lee KJ, Kim EH, Lee HS, Nam CM, Nam HS, Kim YD, Heo JH. Brachial-ankle pulse wave velocity is a strong predictor for mortality in patients with acute stroke. Hypertension 2014; 64: 240-246.

8 Saji N, Kimura K, Kawarai T, Shimizu H, Kita Y. Arterial stiffness and progressive neurological deficit in patients with acute deep subcortical infarction. Stroke 2012; 43: 3088-3090.

9 Saji N, Kimura K, Shimizu H, Kita Y. Association between silent brain infarct and arterial stiffness indicated by brachial-ankle pulse wave velocity. Intern Med 2012; 51: 1003-1008. 
10 Saji N, Shimizu H, Kawarai T, Tadano M, Kita Y, Yokono K. Increased brachial-ankle pulse wave velocity is independently associated with white matter hyperintensities. Neuroepidemiology 2011; 36: 252-257.

11 Saji N, Kimura K, Shimizu H, Kita Y. Silent brain infarct is independently associated with arterial stiffness indicated by cardio-ankle vascular index (CAVI). Hypertens Res 2012; 35: 756-760.

12 Kubota Y, Maebuchi D, Takei M, Inui Y, Sudo Y, Ikegami Y, Fuse J, Sakamoto M, Momiyama Y. Cardio-ankle vascular index is a predictor of cardiovascular events. Artery Res 2011; 5: 91-96.

13 Choi SY, Park HE, Seo H, Kim M, Cho SH, Oh BH. Arterial stiffness using cardio-ankle vascular index reflects cerebral small vessel disease in healthy young and middle aged subjects. J Atheroscler Thromb 2013; 20: 178-185.

14 Shirai K, Hiruta N, Song M, Kurosu T, Suzuki J, Tomaru T, Miyashita Y, Saiki A, Takahashi M, Suzuki K, Takata M. Cardia-ankle vascular index (CAVI) as a novel indicator of arterial stiffness: theory, evidence and perspectives. J Atheroscler Thromb 2011; 18: 924-938.

15 Takaki A, Ogawa H, Wakeyama T, Iwami T, Kimura M, Hadano Y, Matsuda S, Miyazaki Y, Hiratsuka A, Matsuzaki M. Cardio-ankle vascular index is superior to brachial-ankle pulse wave velocity as an index of arterial stiffness. Hypertens Res 2008; 31: 1347-1355.

16 Suzuki J, Sakakibara R, Tomaru T, Tateno F, Kishi M, Ogawa E, Kurosu T, Shirai K. Stroke and cardio-ankle vascular stiffness index. J Stroke Cerebrovasc Dis 2013; 22: 171-175.

17 Saji N, Shimizu H, Kawarai T, Tadano M, Kita Y, Yokono K. Clinical features of a firstever lacunar infarction in Japanese patients: poor outcome in females. J Stroke Cerebrovasc Dis 2011; 20: 231-235.

18 Sen S, Lynch DR Jr, Kaltsas E, Simmons J, Tan WA, Kim J, Beck J, Rosamond W. Association of asymptomatic peripheral arterial disease with vascular events in patients with stroke or transient ischemic attack. Stroke 2009; 40: 3472-3477.

19 Meves SH, Diehm C, Berger K, Pittrow D, Trampisch HJ, Burghaus I, Tepohl G, Allenberg JR, Endres HG, Schwertfeger M, Darius H, Haberl RL, getABI Study Group. Peripheral arterial disease as an independent predictor for excess stroke morbidity and mortality in primary-care patients: 5 -year results of the getABI study. Cerebrovasc Dis 2010; 29: 546-554.

20 Gronewold J, Hermann DM, Lehmann N, Kröger K, Lauterbach K, Berger K, Weimar C, Kälsch HI, Moebus S, Jöckel KH, Bauer M, Erbel R, Heinz Nixdorf Recall Study Investigative Group. Ankle-brachial index predicts stroke in the general population in addition to classical risk factors. Atherosclerosis 2014; 233: 545-550.

21 De Silva DA, Woon FP, Gan HY, Chen CP, Chang HM, Koh TH, Kingwell BA, Cameron JD, Wong MC. Arterial stiffness is associated with intracranial large artery disease among ethnic Chinese and South Asian ischemic stroke patients. J Hypertens 2009; 27: 1453-1458.

22 Zhang J, Li Y, Wang Y, Niu W, Zhang Y, Gao P, Zhang L, Lin H, Chen K, Zhu D. Arterial stiffness and asymptomatic intracranial large arterial stenosis and calcification in hypertensive chinese. Am J Hypertens 2011; 24: 304-309.

$23 \mathrm{Kim}$ J, Cha MJ, Lee DH, Lee HS, Nam CM, Nam HS, Kim YD, Heo JH. The association between cerebral atherosclerosis and arterial stiffness in acute ischemic stroke. Atherosclerosis 2011; 219: 887-891.
24 Adams HP Jr, Bendixen BH, Kappelle LJ, Biller J, Love BB, Gordon DL, Marsh EE 3rd. Classification of subtype of acute ischemic stroke. Definitions for use in a multicenter clinical trial. Toast. Trial of org 10172 in acute stroke treatment. Stroke 1993; 24. $35-41$.

25 Tuttolomondo A, Di Sciacca R, Di Raimondo D, Serio A, D'Aguanno G, Pinto A, Licata G. Arterial stiffness indexes in acute ischemic stroke: relationship with stroke subtype. Atherosclerosis 2010; 211: 187-194.

26 Isa K, Sakima A, Sakima H, Nakachi K, Kinjyo K, Ohya Y. Association between the intima-media thickness of the brachiocephalic trunk and white matter hyperintensity in brain MRI. Hypertens Res 2013; 36: 980-984.

27 Xie W, Liu J, Wang W, Wang M, Li Y, Sun J, Liu J, Qi Y, Zhao F, Zhao D. Five-year change in systolic blood pressure is independently associated with carotid atherosclerosis progression: a population-based cohort study. Hypertens Res 2014; 37: 960-965.

28 Handa N, Matsumoto M, Maeda H, Hougaku H, Kamada T. Ischemic stroke events and carotid atherosclerosis. Results of the Osaka Follow-up Study for Ultrasonographic Assessment of Carotid Atherosclerosis (the OSACA Study). Stroke 1995; 26: 1781-1786.

29 Ochi N, Tabara Y, Igase M, Nagai T, Kido T, Miki T, Kohara K. Silent cerebral microbleeds associated with arterial stiffness in an apparently healthy subject. Hypertens Res 2009; 32: 255-260.

30 Hatanaka R, Obara T, Watabe D, Ishikawa T, Kondo T, Ishikura K, Aikawa T, Aono Y, Hara A, Metoki H, Asayama K, Kikuya M, Mano N, Ohkubo T, Izumi S, Imai Y. Association of arterial stiffness with silent cerebrovascular lesions: the Ohasama study. Cerebrovasc Dis 2011; 31: 329-337.

$31 \mathrm{Kim}$ DH, Kim J, Kim JM, Lee AY. Increased brachial-ankle pulse wave velocity is independently associated with risk of cerebral ischemic small vessel disease in elderly hypertensive patients. Clin Neurol Neurosurg 2008; 110: 599-604.

32 Ohmine T, Miwa Y, Yao H, Yuzuriha T, Takashima Y, Uchino A, Takahashi-Yanaga F, Morimoto S, Maehara Y, Sasaguri T. Association between arterial stiffness and cerebral white matter lesions in community-dwelling elderly subjects. Hypertens Res 2008; 31 . 75-81.

33 Stevenson SF, Doubal FN, Shuler K, Wardlaw JM. A systematic review of dynamic cerebral and peripheral endothelial function in lacunar stroke versus controls. Stroke 2010; 41: e434-e442.

34 Wardlaw JM. Blood-brain barrier and cerebral small vessel disease. J Neurol Sci 2010; 299: 66-71.

35 Hu H, Cui H, Han W, Ye L, Quu W, Yang H, Zhang C, Guo X, Mao G. A cutoff point for arterial stiffness using the cardio-ankle vascular index based on carotid arteriosclerosis. Hypertens Res 2013; 36: 334-341.

36 Horinaka S, Yabe A, Yagi H, Ishimura K, Hara H, lemua T, Matsuoka H. Comparison of atherosclerotic indicators between cardio ankle vascular index and brachial ankle pulse wave velocity. Angiology 2009; 60: 468-476.

37 Kawai T, Ohishi M, Takeya Y, Onishi M, Ito N, Oguro R, Yamamoto K, Kamide K, Rakugi $\mathrm{H}$. Carotid plaque score and intima media thickness as predictors of stroke and mortality in hypertensive patients. Hypertens Res 2013; 36: 902-909.

Supplementary Information accompanies the paper on Hypertension Research website (http://www.nature.com/hr) 